\title{
Submitted: Is contrast-enhanced ultrasonography (CEUS) useful Accepted: 21.06.2021 in the assessment of obstructive uropathy-induced perfusion disorders?
}

Published: 29.11.2021

\begin{abstract}
Keywords
obstructive uropathy,

CEUS,

perfusion disorders
\end{abstract}

\author{
Sergiusz Ustyniak¹, Ilona Kurnatowska², Ludomir Stefańczyk, \\ Magdalena Kaczmarska1', Patryk Wieczorek ${ }^{1}$
}

${ }^{1}$ Department of Radiology and Diagnostic Imaging, Norbert Barlicki Memorial Teaching Hospital No. 1 of The Medical University of Lodz, Poland

${ }^{2}$ Department of Internal Medicine and Transplantation Nephrology, Norbert Barlicki Memorial Teaching Hospital No. 1 of The Medical University of Lodz, Poland

Correspondence: Sergiusz Ustyniak, Department of Radiology and Diagnostic Imaging, Norbert Barlicki Memorial Teaching Hospital No. 1 of The Medical University of Lodz, Poland; e-mail:sergiusz.ustyniak@gmail.com

DOI: $10.15557 / \mathrm{JoU} .2021 .0057$

\begin{abstract}
Aim: The study aimed to evaluate the usefulness of contrast-enhanced ultrasonography in renal perfusion disorders caused by obstructive uropathy. Case description: We present a case of a patient with a horseshoe kidney, in which the urine outflow from the right part of the kidney was blocked due to the presence of a bladder tumour infiltrating the ureter. Perfusion disorders in both parts of the kidney, visible in computed tomography, were partially reflected in the ultrasound image. Conclusions: Doppler ultrasound and contrast-enhanced ultrasound may be useful for monitoring perfusion disorders in the course of obstructive uropathy, provided that appropriate methodology is developed to compare blood flow in both kidneys.
\end{abstract}

\section{Introduction}

Contrast-enhanced ultrasound (CEUS) is increasingly used in clinical practice. Apart from its application in the assessment of the liver and the spleen, the method is also used in the diagnosis of renal pathology ${ }^{(1-6)}$.

\section{Case report}

A 74-year-old patient burdened with multiple medical conditions (obesity, type 2 diabetes, myocardial infarction and chronic kidney disease with creatinine levels of approximately $1.6 \mathrm{mg} \%$; eGFR $30 \mathrm{~mL} / \mathrm{min} / 1.73 \mathrm{~m}^{2}$ for several years) was admitted to the surgical department due to abdominal pain and general weakness. Abdominal tenderness, guarding and resistance in the right iliac fossa were found on palpation. Renal function was impaired (creatinine levels of $3.2 \mathrm{mg} \%$, eGFR $13 \mathrm{~mL} / \mathrm{min} / 1.73 \mathrm{~m}^{2}$, urea $122 \mathrm{mg} \%$, no electrolyte disorders, CRP $189 \mathrm{mg} / \mathrm{L}$
(N: 0-5 mg/L) with preserved diuresis (about 1.5 litres per day). Ultrasound (GE Logiq S8, USA) revealed a horseshoe kidney and a dilation of the right renal pelvis to $25-30 \mathrm{~mm}$. In addition, there was a small calculus in the left part of the kidney. The evaluation of the urinary bladder was difficult due to the presence of a Foley catheter.

On the next day, a multiphase CT scan (GE Revolution CT, USA) of the abdomen was performed. Fat stranding was found around the aorta, the origin of the mesenteric artery and accessory renal arteries. The horseshoe kidney was placed low - the isthmus was below the inferior mesenteric artery origin. The right renal pelvis was dilated to $29 \times 37 \mathrm{~mm}$. The right side of the kidney showed weaker contrast enhancement (Fig. 1 and Fig. 2). Several calculi were found in the left part of the kidney (Fig. 3 and Fig. 4). In the right part of the urinary bladder there was a nonenhancing lesion. Additionally, colonic diverticulosis was found. On day 4 of hospitalization, renal CEUS was performed. $4.8 \mathrm{~mL}$ of SonoVue was administered as a single 


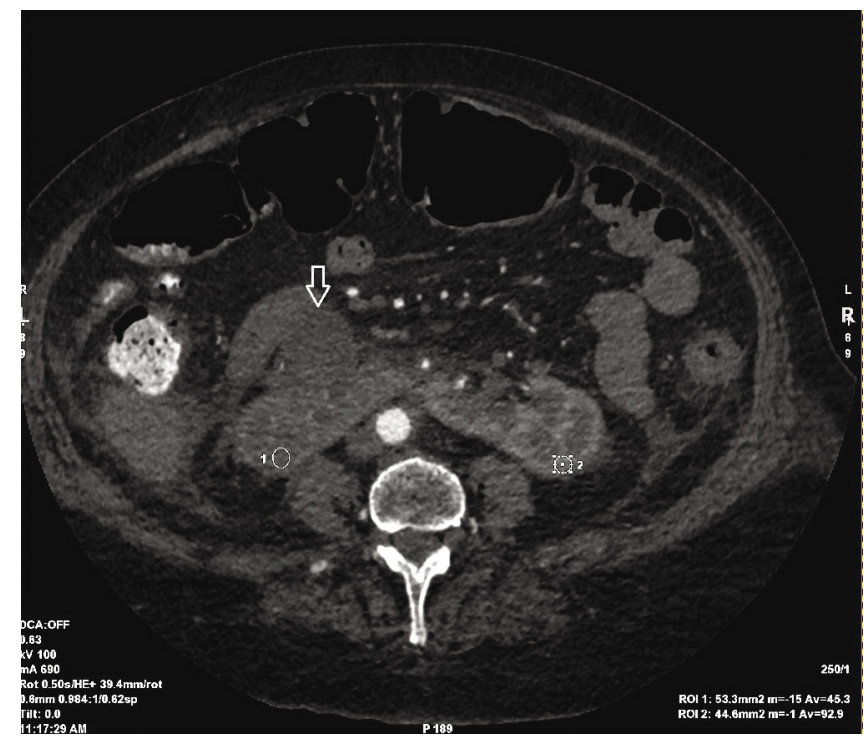

Fig. 1. CT image of the abdominal cavity in the arterial phase (acquisition approximately 20 seconds after intravenous injection of contrast agent) - clearly weaker enhancement of the cortex of the right part of the horseshoe kidney (45 vs $93 \mathrm{HU}$ ). The pelvicalyceal system of the right part of the kidney is widened (arrow)

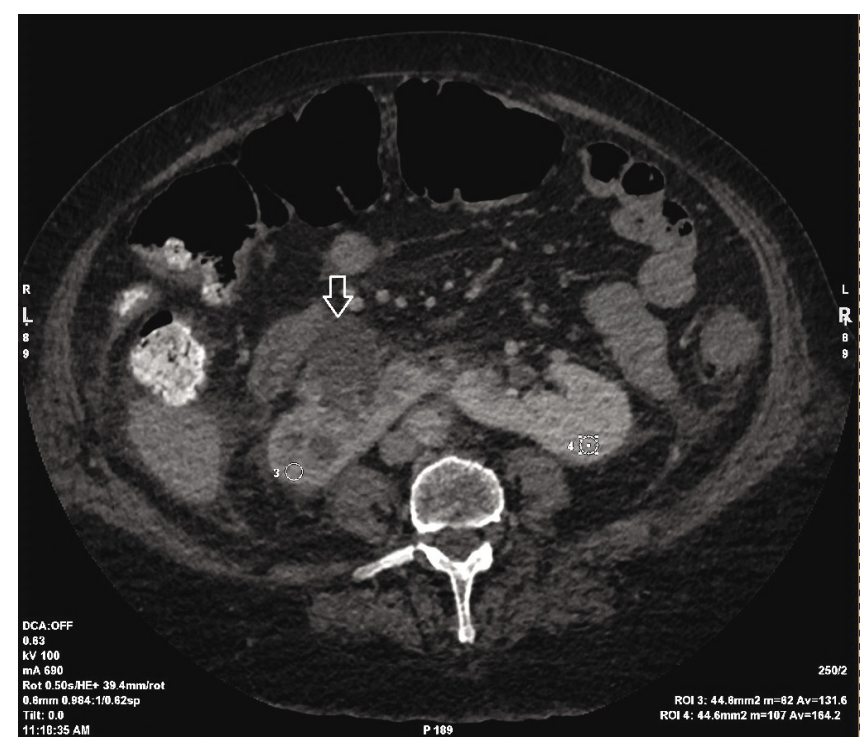

Fig. 2. CT image of the abdominal cavity in the parenchymal phase (acquisition approximately 50 seconds after intravenous injection of contrast agent) - weaker enhancement of the cortex of the right part of the horseshoe kidney persists (132 vs 164 $H U$ ). Visible dilatation of the right pelvis of the kidney (arrow)

bolus with saline flush intravenously (Bracco, Italy). There was a slight further dilation of the pelvis in the right part of the kidney $(29 \times 39 \mathrm{~mm})$. Doppler ultrasound showed increased flow resistance in the corticomedullary border to 0.76 on the right and 0.72 on the left. The enhancement curves from both parts of the kidney were similar with a slightly delayed inflow and outflow of contrast in the right part. The renal pelvis did not enhance and was used as a reference point (Fig. 5, Fig. 6, Fig. 7). The urinary bladder remained difficult to fully evaluate. The patient was

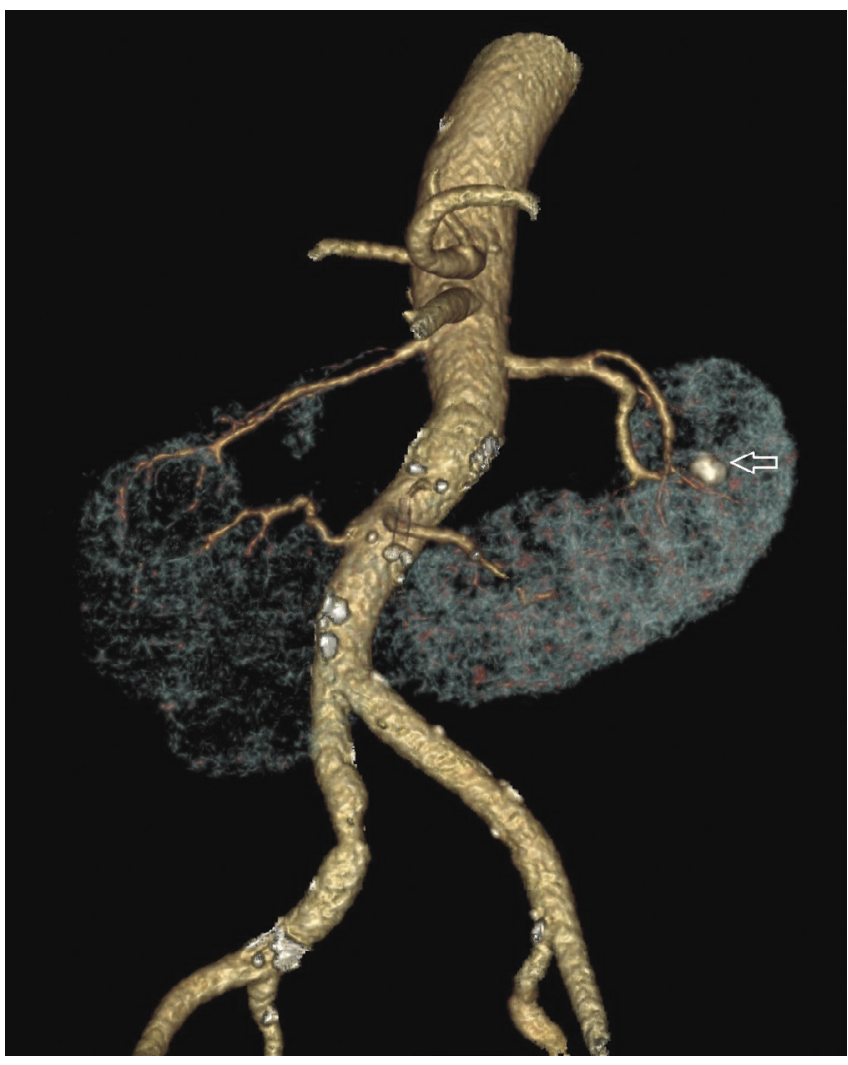

Fig. 3.3 reconstruction of the CT image of the abdominal cavity in the arterial phase-weaker enhancement of the cortex of the right part of the horseshoe kidney. A calculus in the left part of the kidney (arrow). 4 renal arteries supply the kidney

treated conservatively (fluid therapy, broad-spectrum antibiotic therapy, thromboprophylaxis, insulin therapy) with a slight improvement of renal function (eGFR $28 \mathrm{~mL} /$ $\min / 1.73 \mathrm{~m}^{2}$ ). On day 10 , the patient was transferred to the Urology Department. Endoscopy of the urinary bladder showed a tumour infiltrating the ureter. A ureteral catheter (Double J) was inserted into the renal pelvis on the right side, which restored urinary outflow and resulted in clinical improvement. The patient refused surgical treatment for a bladder tumour and still remains under observation.

\section{Discussion}

In addition to morphological evaluation, renal blood flow and glomerular filtration (GFR) are assessed to monitor pathology, regardless of the underlying cause. The possibilities of assessing renal perfusion in US, CT and MRI are not fully understood and validated ${ }^{(1,7-9)}$. Contrast-enhanced $\mathrm{CT}$ is widely used in this area. However, a CT scan involves exposure to radiation (which is especially important in multiphase protocols) and administration of a nephrotoxic iodine contrast agent, therefore it cannot always be performed $^{(5)}$.

Reduced renal blood flow and glomerular filtration rate manifest in a CT image as weaker enhancement of the renal parenchyma ${ }^{(8,9)}$. Obstruction of urine outflow results 


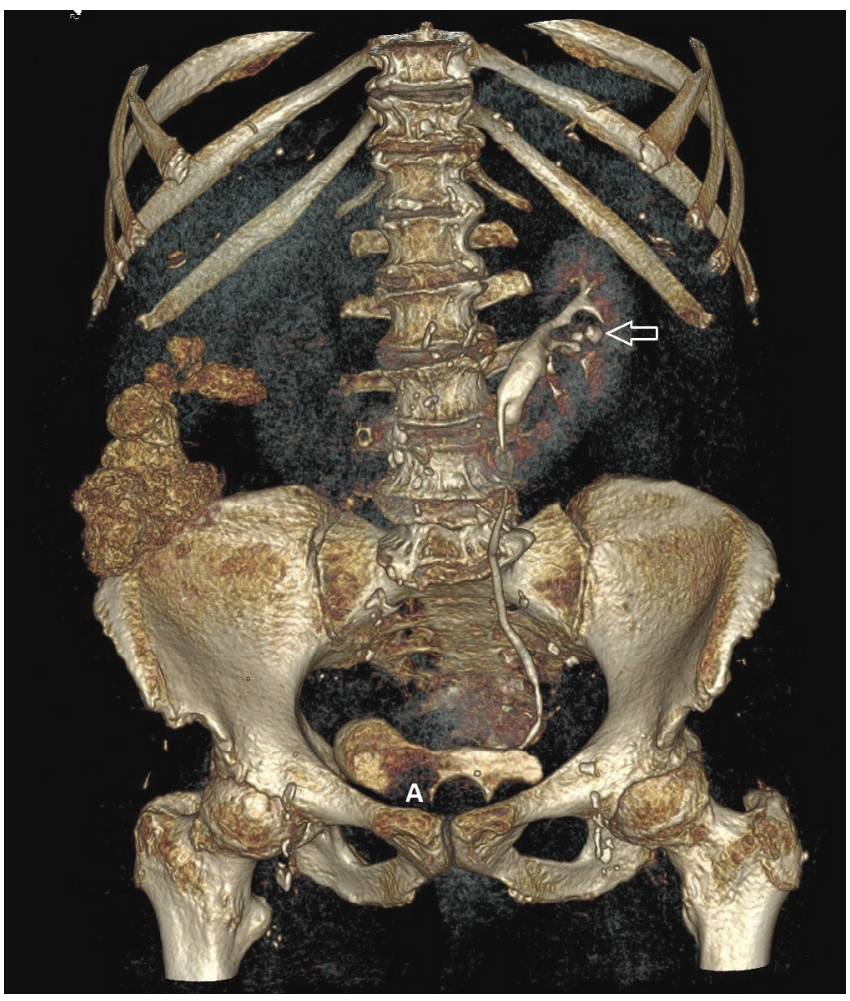

Fig. 4. CT image of the abdominal cavity in the excretory phase (acquisition approximately 7 minutes after intravenous injection of contrast agent) $-A$ d reconstruction shows lack of urine excretion from the right part of the kidney, left pelvicalyceal system enhances strongly. The non-enhancing region in the bladder corresponds to a tumour infiltrating the right ureter

in weaker contrast enhancement of the kidney and delayed contrast agent passage into the urine (without the possibility of precise quantification $)^{(3,9)}$. Renal artery stenosis also results in a reduced enhancement of the renal parenchyma, with the difference that in the arterial phase it is usually possible to quantify the degree of stenosis, and in the excretory phase there is a discernible passage of the contrast agent into the urine (without a possibility of accurate quantification). Atherosclerotic calcifications or aortic stents may impede the assessment of arterial stenosis ${ }^{(1,8)}$. There are situations when weaker kidney contrast enhancement is an incidental finding and a multiphase exam is not available. In the described case, weaker enhancement of the right part of the kidney was found in all phases of CT, renal artery stenosis was excluded and no contrasting urine was excreted from the right part of the kidney, which suggested the diagnosis of obstructive uropathy.

Contrast-enhanced ultrasonography is a recognized method for the assessment of kidney lesions and perfusion disorders resulting from impaired patency of the renal vessels ${ }^{(2-4,10-12)}$. Modern ultrasonography contrast agents persist in the vessels, without interstitial diffusion and glomerular filtration. They are useful in the assessment of renal perfusion. The use of modern ultrasonography in the assessment of glomerular filtration disorders is difficult to validate because of the method limitations ${ }^{(1)}$. CEUS is based on nonlinear echo amplification, which complicates the necessary image correction resulting from anatomical conditions and the characteristics of the tissues in the examined area. In this case, ultrasound attenuation cannot be compensated with standard algorithms such as time gain compensation. Moreover, there is no simple relationship between the concentration of the agent and the echo intensity and some microbubbles are degraded before they reach the assessed $\operatorname{organ}^{(1,5)}$. Kidney enhancement curves represent the influx peak followed by successive waves of recirculation. During the examination, only one kidney is imaged, which makes it difficult to compare the degree of enhancement of one

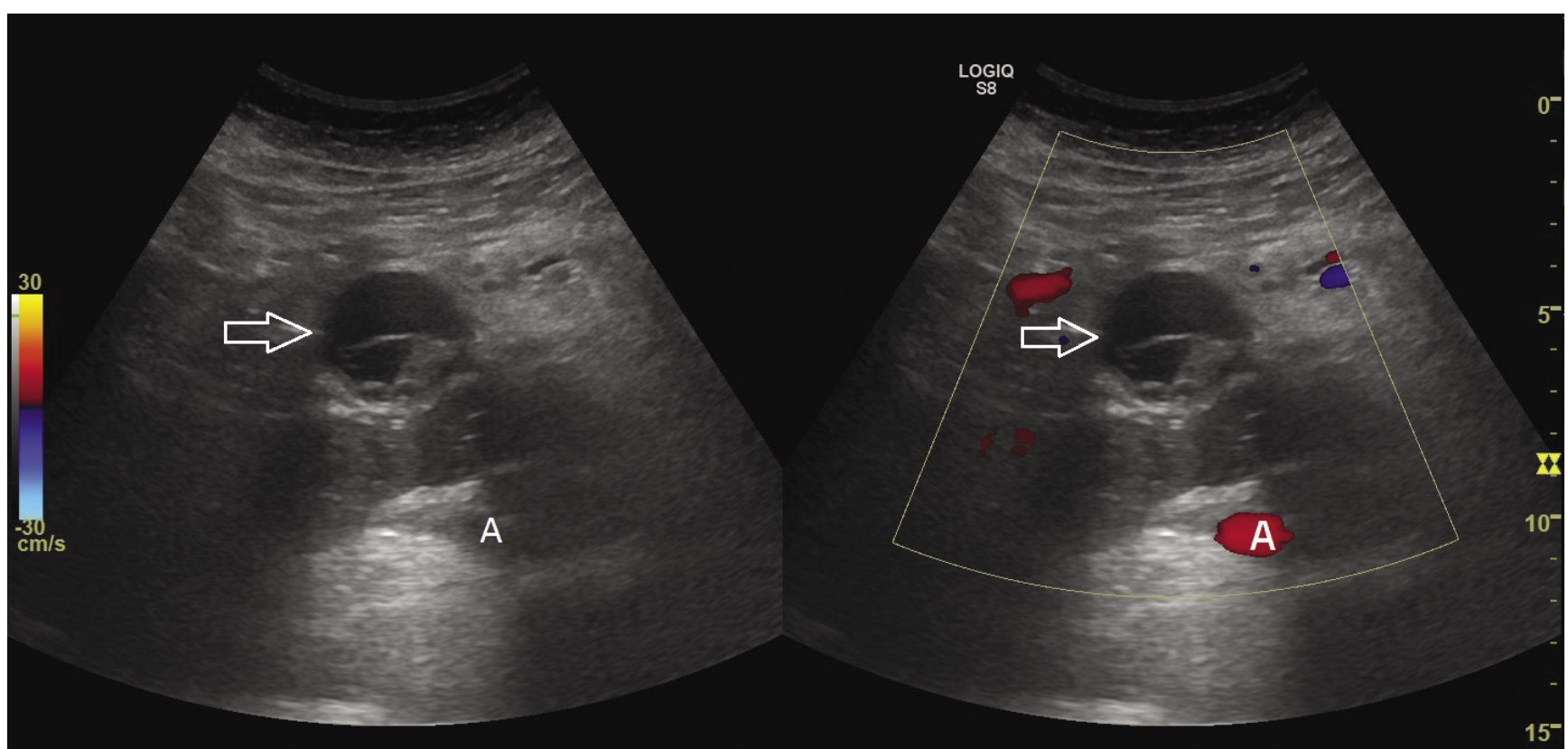

Fig. 5. Axial ultrasound image of the umbilical region. Visible dilatation of the right pelvis of the kidney (arrow). The location of the aorta was marked (A) 


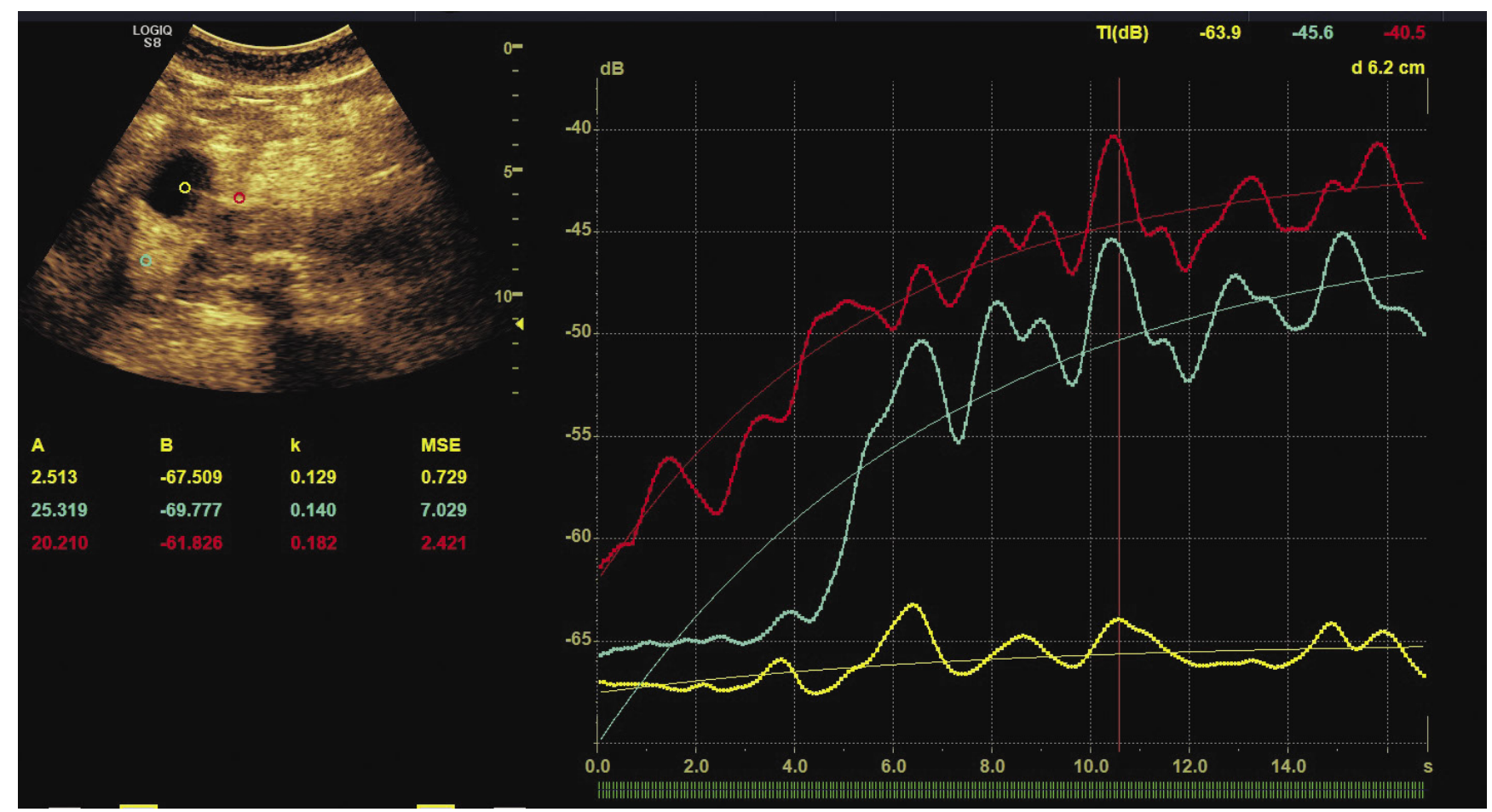

Fig. 6. Axial CEUS image of the umbilical region - the enhancement curves from both parts of the kidney are similar in shape-slightly delayed inflow of contrast to the deeper right part of the kidney (blue vs red curve). The dilated renal pelvis (reference point - yellow curve) is not enhanced

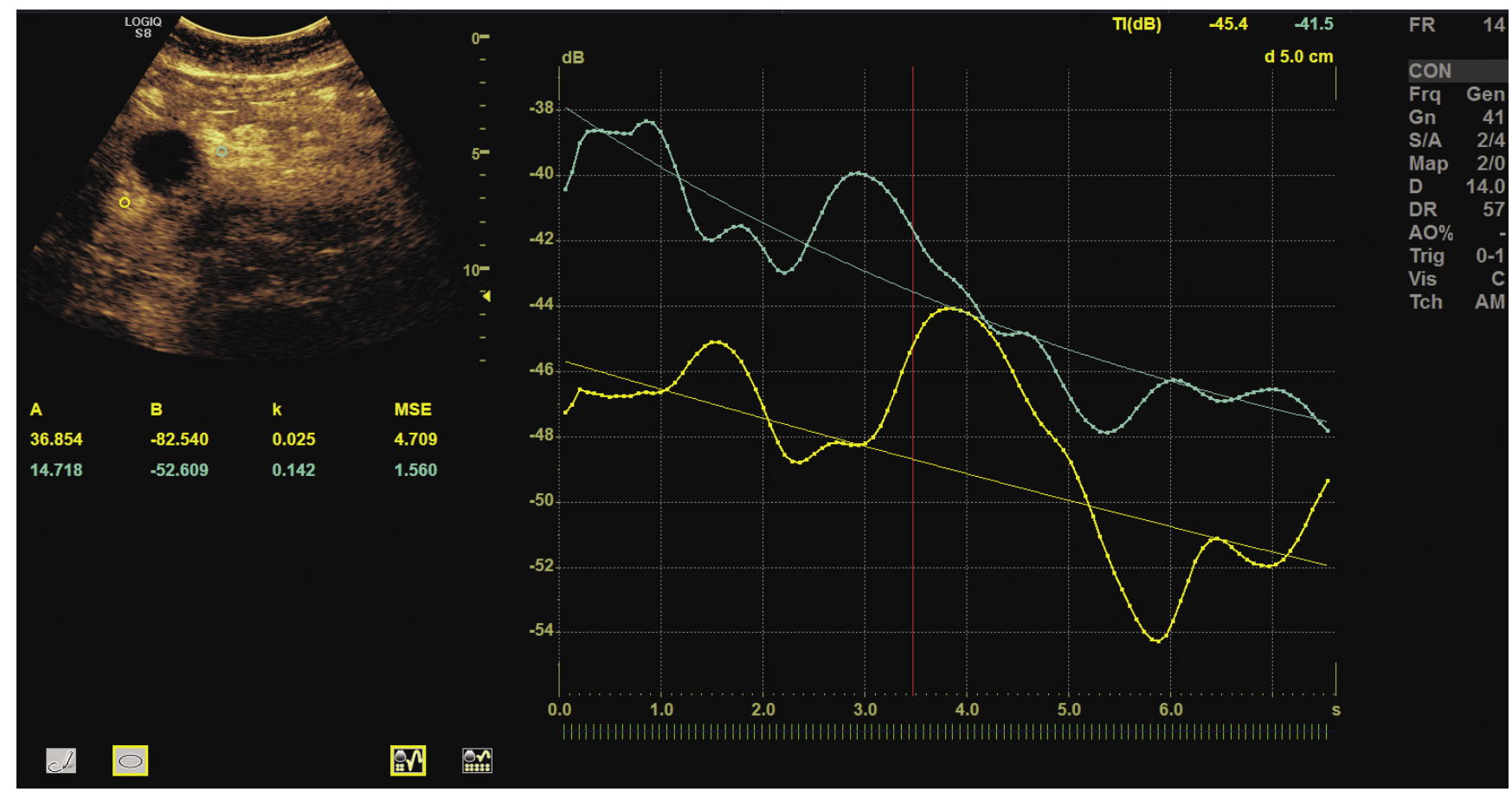

Fig. 7. An axial CEUS image of the umbilical region in the late phase - the enhancement curves from both parts of the kidney are similar in shape same as in the early stages - slightly delayed outflow of contrast to the deeper right part of the kidney (blue vs yellow curve)

kidney with the another one and assess the influence of systemic conditions. Despite the described problems with the validation of CEUS, it was possible to establish that renal artery stenosis extends the time of contrast flow into the kidney ${ }^{(4)}$. Prolongation of the acceleration phase and flattening of the flow spectrum curve in patients with renal arterial stenosis are observed in Doppler examinations ${ }^{(10)}$. It has been already proven that reduced perfusion due to obstruction of urine outflow from the pelvicalyceal system increases the peripheral resistance in the vascular 
bed of the kidney, which is reflected in an increase in the resistive index $(\mathrm{RI})$, while the acceleration phase remains normal $^{(11,12)}$. Doppler exam complements the information obtained from the CEUS enhancement curve analysis. However, it should be noted that the flow spectrum registration in the kidney using the Doppler technique is not always possible, especially in obese or severely ill people $\mathrm{e}^{(3)}$.

The unique anatomical situation of the horseshoe kidney with partial obstruction of the pelvicalyceal system allowed for a simultaneous assessment of both parts of the kidney and the propagation of the contrast agent.

The degree of parenchymal enhancement in CEUS is similar in both parts of the kidney and the shape of both curves is similar, presenting no changes characteristic of arterial

\section{References}

1. Grenier N, Cornelis F, Le Bras Y, Rigou G, Boutault JR, Bouzgarrou M: Perfusion imaging in renal diseases. Diagn Interv Imaging 2013; 94: 1313-1322.

2. Cokkinos DD, Antypa E, Kalogeropoulos I, Tomais D, Ismailos E, Matsiras I et al.: Contrast-enhanced ultrasound performed under urgent conditions. Indications, review of the technique, clinical examples and limitations. Insights Imaging 2013; 4: 185-198.

3. Mastrorosato M, Bertelli E, Bonini MC, Danti G, Vannini C, Agostini S et al.: Rare case of an upper urinary tract carcinoma (UTUC) in renal pelvis and ureter associated to renal vein thrombosis: diagnostic imaging with CECT, MRI and CEUS. J Ultrasound 2019; 22: 371.

4. Ren JH, Ma N, Wang SY, Sun YJ, Zhang YW, Guo FJ et al.: Rationale and study design for one-stop assessment of renal artery stenosis and renal microvascular perfusion with contrast-enhanced ultrasound for patients with suspected renovascular hypertension. Chin Med J (Engl) 2019; 132: 63-68.

5. Harvey CJ, Alsafi A, Kuzmich S, Ngo A, Papadopoulou I, Lakhani A et al.: Role of US contrast agents in the assessment of indeterminate solid and cystic lesions in native and transplant kidneys. Radiographics 2015; 35: 1419-1430.

6. Sidhu PS, Cantisani V, Dietrich CF, Gilja OH, Saftoiu A, Bartels E et al.: The EFSUMB guidelines and recommendations for the clinical practice of contrast-enhanced ultrasound (CEUS) in Non-Hepatic Applications: Update 2017 (Long Version). Ultraschall Med 2018; 39: e2-e44. stenosis $^{(5)}$. However, there were discernible differences in the value of renal arterial resistive index. Practical use of these observations can be made if an appropriate protocol with a reliable reference point (i.e. a second kidney) is established or if CEUS is complementary to a CT scan. The exam can also be helpful in the assessment of perfusion disorders due to obstructive uropathy in the situation of initially impaired renal function, which limits the use of contrast-enhanced CT and MRI.

\section{Conflict of interest}

Authors do not report any financial or personal connections with other persons or organizations, which might negatively affect the contents of this publication and/or claim authorship rights to this publication.

7. Jiang K, Ferguson CM, Abumoawad A, Saad A, Textor SC, Lerman LO: A modified two-compartment model for measurement of renal function using dynamic contrast-enhanced computed tomography. PLoS One 2019; 14: e0219605.

8. Kwon SH, Saad A, Herrmann SM, Textor SC, Lerman LO: Determination of single-kidney glomerular filtration rate in human subjects by using CT. Radiology 2015; 276: 490-498.

9. Cai XR, Zhou QC, Yu J, Feng YZ, Xian ZH, Yang WC et al.: Assessment of renal function in patients with unilateral ureteral obstruction using whole-organ perfusion imaging with 320-detector row computed tomography. PLoS One 2015; 10: e0122454.

10. Grzelak P, Szymczyk K, Strzelczyk J, Kurnatowska I, Sapieha M, Nowicki $\mathrm{M}$ et al.: Perfusion of kidney graft pyramids and cortex in contrast-enhanced ultrasonography in the determination of the cause of delayed graft function. Ann Transplant 2011; 16: 48-53.

11. Eryildirim B, Şahan A, Türkoğlu Ö, Tuncer M, Kavukoğlu Ö, Şimsek B et al.: Non-invasive evaluation of obstruction after ureteroscopic stone removal: role of renal resistive index assessment. Arch Ital Urol Androl 2020; 92.

12. Miletić D, Fuckar Z, Sustić A, Mozetic V, Smokvina A, Stancić M: Resistance and pulsatility indices in acute renal obstruction. J Clin Ultrasound 1998; 26: 79-84. 